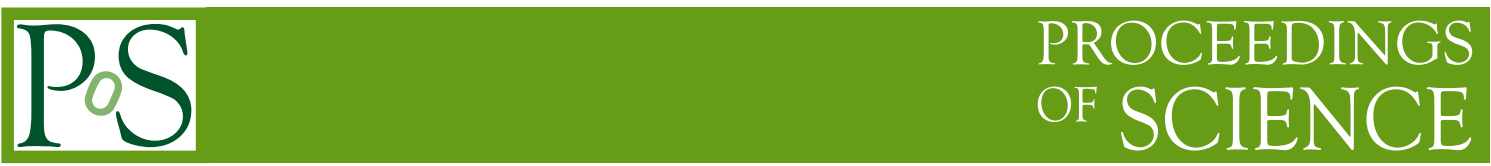

\title{
Commissioning and Plans for the LHCb Trigger
}

\author{
Leandro de Paula*, on behalf of the LHCb Collaboration \\ Universidade Federal do Rio de Janeiro \\ E-mail: leandro.de.paula@cern.ch
}

The $\mathrm{LHCb}$ detector has been designed to study with high precision rare $b$-hadron decays produced at the LHC. The LHCb trigger is of crucial importance in selecting the collisions of interest and is based on a two-level system. The first level, Level 0, is implemented in hardware and uses information from the calorimeter, muon and pile-up systems to select events containing particles with large transverse momentum while rejecting events with multiple interactions. The Level 0 trigger accepts events at a rate of $1 \mathrm{MHz}$ with which the detector information is read out and fed into the High Level Trigger. This software trigger has an output rate of $2 \mathrm{kHz}$ and takes the decisions based on fully reconstructed events. The trigger commissioning and its expected performance for some scenarios are discussed.

12th International Conference on B-Physics at Hadron Machines - BEAUTY 2009

September 07 - 112009

Heidelberg, Germany

\footnotetext{
* Speaker.
} 


\section{Introduction}

The LHCb detector [1] is a single arm spectrometer that has been constructed to exploit the large $b \bar{b}$-pairs production at the LHC, in order to make precise studies of rare b-hadron decays. According to the design, proton beams will collide at the LHCb interaction point with $14 \mathrm{TeV}$ center of mass energy and an average luminosity of $2 \times 10^{32} \mathrm{~cm}^{-2} \mathrm{~s}^{-1}$. Under these conditions the mean number of collisions per bunch crossing is close to one. Due to the LHC bunch structure and the detector geometrical acceptance the frequency of bunch crossings with visible interactions will be about $10 \mathrm{MHz}$.

The fraction of visible events in which $b$-quarks are produced will be about $1 \%$, only a fraction of them will have all the decay products of at least one $b$-hadron reconstructed. Furthermore the branching ratios of $\mathrm{B}$-mesons used to study $\mathrm{CP}$ violation are typically less than $10^{-3}$. In order to enhance the signal to back-ground ratio the event selections will have to exploit the relatively large $b$ mass and lifetime applying stringent cuts.

One of the challenging requirements of $\mathrm{LHCb}$ is to reduce the interaction rate down to the 2 $\mathrm{kHz}$ allowed by the long-term data storage resources, while providing good efficiency for relevant channels. This can be accomplished using the $\mathrm{b}$ main signatures of $b$-hadron decays: relatively high transverse momentum $\left(p_{T}\right)$ and large impact parameter (IP) with respect to the proton-proton interaction vertex.

\section{Trigger Architecture and Implementation}

The LHCb trigger is composed of two levels, called Level 0 (L0) and High Level Trigger (HLT). L0 is a synchronous system with fixed latency, implemented in custom electronics. It reduces the rate from the interaction rate $(10 \mathrm{MHz})$ to $1 \mathrm{MHz}$. At this frequency the whole detector can be read-out and the HLT can take the final decision based on the full detector information. The HLT algorithms will run on about 1500 double quad-core computing nodes forming the Event Filter Farm (EFF). The output rate is limited by the logging bandwidth to $2 \mathrm{kHz}$. In parallel to the trigger lines that are presented in this section there are trigger lines dedicate to monitoring and calibration that will not be discussed in this article.

\subsection{L0 Trigger}

In order to identify events with large $\mathrm{p}_{\mathrm{T}}$ products, L0 uses information from selected subdetectors which are read-out at $40 \mathrm{MHz}$. Three types of $\mathrm{L} 0$-objects are built from the sub-detector information and transferred to the L0 Decision Unit (LODU) to take the trigger decision:

- L0 Hadron - The highest transverse energy deposition found in the hadron calorimeter is transferred to the LODU.

- L0 Electromagnetic Cluster - The highest transverse energy deposition measured in the electromagnetic calorimeter for neutral and charged showers are transferred to the LODU.

- L0 Muon - The highest transverse momenta values associated to muon candidates are also transferred to the LODU. 
A positive decision is taken by the LODU when a L0-object above a defined threshold is found. In addition, a fast estimation of the number of proton-proton interaction per bunch crossing can be provided by two dedicated layers of the vertex detector and can be used by the LODU to veto events with more than one interaction.

\subsection{The High Level Trigger}

The HLT has access to information from the whole detector, however a complete event reconstruction is not possible at $1 \mathrm{MHz}$. The HLT is therefore divided in two sequential steps. The first step (HLT1) reduces the rate to allow full reconstruction, which is performed during the second step (HLT2).

Depending on the type of the L0 decision the event is passed to different selection line of the HLT1. The general strategy is to refine the $\mathrm{p}_{\mathrm{T}}$ measurement of the L0 trigger by matching the corresponding L0 objects to track segments in the Tracking Stations or in the Vertex Locator, reaching a momentum resolution of $3 \%$. For the confirmed L0 candidate, the track impact parameter with respect to the primary vertex can be determined and used to reject events. Using additional tracks secondary vertices are searched for. Cuts on the two track invariant mass are applied to reject background. There are five HLT1 lines:

- Hadron Line. Using L0 Hadron candidates as seeds, an event is confirmed if a reconstructed hadron has a transverse momentum ( $\mathrm{p}_{\mathrm{T}}$ ) and an impact parameter (IP) above a given threshold. The event can also be accepted if the L0 seed together with a second reconstructed track form a common secondary vertex.

- Muon Line. L0-Muon or L0-DiMuon objects are used as seeds and confirmed by track segments fulfilling the momentum requirements. The muon line allows to accept events without an IP requirement, only by applying strict $\mathrm{p}_{\mathrm{T}}$ and invariant mass thresholds.

- Muon and Track Line. Events are accepted if it is possible to produce a secondary vertex from a confirmed L0-Muon candidate and a reconstructed track that fulfills $\mathrm{p}_{\mathrm{T}}$, IP and, together with the first track, the invariant mass requirement.

- Electron Line. Starting with an L0-electron candidate this line first confirms the electron and then looks either for a track with large IP and $\mathrm{p}_{\mathrm{T}}$ or for two tracks with a common displaced secondary vertex to accept the event.

- Photon Line. Starting with an L0 electromagnetic energy cluster candidate the algorithm confirms the photon and does the selection using requirements similar to the used in the electron line.

In HLT2 the full event is reconstructed and particle identification information from the calorimeter and the muon system is added. The trigger decision is based on two different types of selections:

- Inclusive selections.

- Topological trigger. If a displaced secondary vertex with an invariant mass of the tracks compatible with a $b(c)$-hadron decay is found, the event is accepted. 


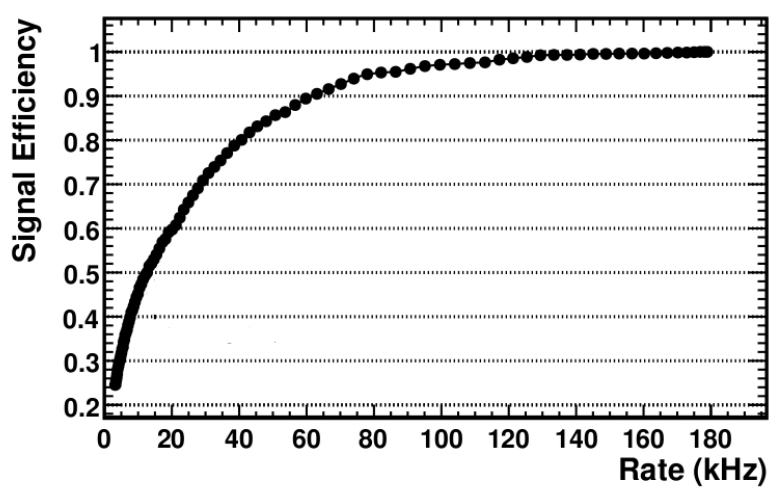

Figure 1: Trigger efficiency (w/r to the offline selection) for the decay $B_{S} \rightarrow J / \Psi \Phi$ and the expected trigger rate when the HLT cut on the muon transverse momentum is varied.

- Inclusive $\phi$ trigger. If a $\phi \rightarrow K^{+} K^{-}$decay is reconstructed and the $\phi$ is not pointing to the primary vertex the event is triggered.

- Leptonic triggers. Either one lepton fulfilling tight $\mathrm{p}_{\mathrm{T}}$ or IP requirements, or a two track vertex formed from at least one lepton fulfilling the invariant mass or IP requirements must be present to accept the event.

- Exclusive Selections. Exclusive decay channels are triggered if identified after full reconstruction.

\subsection{Trigger Implementation}

For the relevant B decay channels one should identify the most performing trigger lines and, for each line, the parameters that can be used to control the minimum bias rate while maintaining an acceptable signal efficiency. As the trigger should be optimised for the events that are used in the physics analysis, the efficiencies are calculated with respect to the off-line selected event samples.

As an example Fig 1 shows for the decays $B_{s} \rightarrow J / \Psi \phi$ the signal efficiency (with respect to the offline selection) and the expected trigger rate when the HLT cut on the muon transverse momentum is varied. The optimization procedure should keep the redundancy of different selections and should avoid to introduce acceptance cuts that will bias the relevant exclusive quantities.

\section{Bandwidth Division}

The total available bandwidth of the LHCb Trigger/DAQ system is limited, as explained in section 2 , and has to be shared by all trigger lines. The bandwidth division among the trigger lines is established through an optimisation procedure that uses a set of selected physical decay channels (BW-Channels). The optimisation first determines the best efficiency which can be obtained for a BW-Channel for a certain trigger line by varying all the cuts, while constraining the trigger rate to the allowed bandwidth. In a second iteration all BW-Channel efficiencies are optimised simultaneously, assuring that for each channel the efficiency loss relative to its maximum efficiency 


\begin{tabular}{|c|c|c|c|c|c|c|}
\hline L0 & Hadron & \multicolumn{2}{|c|}{ Muon } & \multicolumn{2}{|c|}{ Electromag. } & Combined \\
\hline rate $(\mathrm{kHz})$ & 630 & \multicolumn{2}{|c|}{280} & \multicolumn{2}{|c|}{180} & 1000 \\
\hline \hline L0 $\times \mathrm{HLT} 1$ & $\mathrm{~h}$ & $\mu$ & $\mu+$ Track & $e$ & $\gamma$ & \\
\hline rate $(\mathrm{kHz})$ & 17 & 13 & 2 & 6 & 8 & 38 \\
\hline
\end{tabular}

Table 1: Rates for different L0 and LO $\times$ HLT1 decisions. L 0 and HLT1 lines are described in the text.

\begin{tabular}{|l|c|c|c|c|c|}
\hline & b-Topological & c-Topological & Inclusive $\Phi$ & Lepton & Combined \\
\hline rate $(\mathrm{Hz})$ & 650 & 200 & 150 & 900 & 1800 \\
\hline
\end{tabular}

Table 2: Rates for $\mathrm{L} 0 \times$ HLT $1 \times$ HLT2(inclusive) decisions.

\begin{tabular}{|c|c|c|c|c|c|c|c|}
\hline \multirow{2}{*}{ Channel } & \multirow{2}{*}{$\begin{array}{c}\text { L0 } \\
\text { HLT1 }\end{array}$} & \multirow{2}{*}{$\begin{array}{c}\text { Hadron } \\
h\end{array}$} & \multicolumn{2}{|c|}{ Muon } & \multicolumn{2}{|c|}{ Electromag. } & \multirow{2}{*}{$\begin{array}{c}\text { Total } \\
\text { Efficiency }\end{array}$} \\
\hline & & & $\mu$ & $\mu+\mathrm{Tr}$. & $e$ & $\gamma$ & \\
\hline \multirow{2}{*}{$B_{d} \rightarrow K^{*} \mu \mu$} & L0 & $31 \%$ & \multicolumn{2}{|c|}{$84 \%$} & \multicolumn{2}{|c|}{$7 \%$} & $88 \%$ \\
\hline & L0 $\times$ HLT1 & $17 \%$ & $77 \%$ & $73 \%$ & $1 \%$ & $2 \%$ & $83 \%$ \\
\hline \multirow{2}{*}{$B_{s} \rightarrow J / \Psi \Phi$} & L0 & $27 \%$ & \multicolumn{2}{|c|}{$91 \%$} & \multicolumn{2}{|c|}{$8 \%$} & $92 \%$ \\
\hline & L0 $\times$ HLT1 & $7 \%$ & $84 \%$ & $62 \%$ & $1 \%$ & $1 \%$ & $86 \%$ \\
\hline \multirow{2}{*}{$B_{s} \rightarrow \mu \mu$} & L0 & $17 \%$ & \multicolumn{2}{|c|}{$98 \%$} & \multicolumn{2}{|c|}{$5 \%$} & $98 \%$ \\
\hline & L0×HLT1 & $3 \%$ & $97 \%$ & $91 \%$ & $0 \%$ & $2 \%$ & $97 \%$ \\
\hline \multirow{2}{*}{$B \rightarrow h h$} & L0 & $60 \%$ & \multicolumn{2}{|c|}{$10 \%$} & \multicolumn{2}{|c|}{$10 \%$} & $65 \%$ \\
\hline & L0 $\times$ HLT1 & $49 \%$ & $4 \%$ & $2 \%$ & $4 \%$ & $1 \%$ & $52 \%$ \\
\hline \multirow{2}{*}{$B_{u} \rightarrow D^{0}\left(K_{s} \pi \pi\right) K$} & L0 & $50 \%$ & \multicolumn{2}{|c|}{$11 \%$} & \multicolumn{2}{|c|}{$7 \%$} & $55 \%$ \\
\hline & L0×HLT1 & $31 \%$ & $6 \%$ & $4 \%$ & $2 \%$ & $2 \%$ & $36 \%$ \\
\hline \multirow{2}{*}{$B_{s} \rightarrow \Phi \gamma$} & L0 & $50 \%$ & \multicolumn{2}{|c|}{$10 \%$} & \multicolumn{2}{|c|}{$72 \%$} & $82 \%$ \\
\hline & L0×HLT1 & $12 \%$ & $4 \%$ & $1 \%$ & $1 \%$ & $51 \%$ & $58 \%$ \\
\hline
\end{tabular}

Table 3: L0 and L0 $\times$ HLT1 Efficiencies for B Channels.

\begin{tabular}{|c|c|c|c|c|}
\hline Channel & b-topological & inclusive $\Phi$ & leptonic & combined \\
\hline$B_{d} \rightarrow K^{*} \mu \mu$ & $59 \%$ & - & $70 \%$ & $74 \%$ \\
\hline$B_{s} \rightarrow J / \Psi \Phi$ & $34 \%$ & $38 \%$ & $82 \%$ & $84 \%$ \\
\hline$B_{s} \rightarrow \mu \mu$ & $80 \%$ & - & $94 \%$ & $95 \%$ \\
\hline$B \rightarrow h h$ & $42 \%$ & - & $2 \%$ & $42 \%$ \\
\hline$B_{u} \rightarrow D^{0}\left(K_{s} \pi \pi\right) K$ & $18 \%$ & - & $4 \%$ & $21 \%$ \\
\hline$B_{s} \rightarrow \Phi \gamma$ & $1 \%$ & $50 \%$ & $1 \%$ & $51 \%$ \\
\hline
\end{tabular}

Table 4: L $0 \times$ HLT $1 \times$ HLT2 Efficiencies for BW-Channels for relevant trigger lines.

obtained in the first step is minimised. Table 1 gives the result obtained for the bandwidth division among L0 and HLT1 lines and table 2 presents the result of the procedure when applied to the HLT2 inclusive lines. The efficiencies calculated with respect to offline selected events for the BW-Channels are presented in tables 3 and 4.

After the trigger thresholds have been set the efficiencies for other channels are checked. Figure 2 shows the resulting L0 and combined L $0 \times$ HLT1 efficiencies for several relevant channels. 

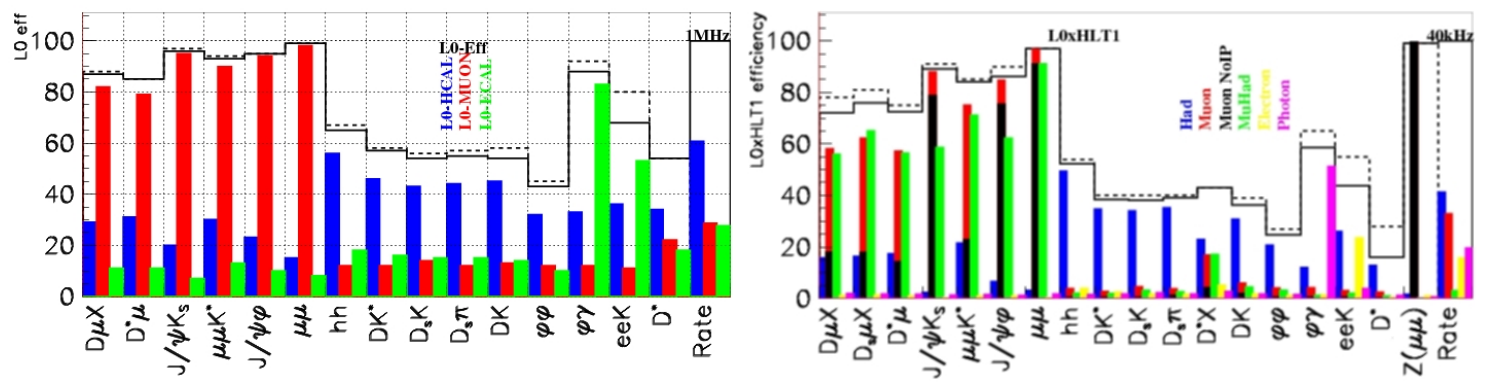

Figure 2: The left (right) plot shows the L0 (combined L0 $\times$ HLT1) efficiencies for off-line selected events for a set of relevant channels. In both plots the doted line indicates the maximum efficiency that can be reached for each channel, supposing that all the bandwidth is given to that BW-Channel. The solid line is the efficiency obtained through the optimisation procedure described in the text. The vertical bars show the efficiency of individual trigger decisions for each channel. Both plots indicates the corresponding trigger rates.

\section{2010 Running Conditions and Trigger Commissioning}

The priority during the first period of data taking is to collect a sample of $10^{8}$ minimum bias events, which can be achieved requesting only some energy deposition in the calorimeter. This sample will be used to perform flavour production studies. Then L0, HLT1 and HLT2 will be gradually put into operation. As the 2010 LHC running conditions are not yet completely established, the trigger has to be ready for several scenarios but in order to simplify the off-line analysis, a minimum number of different settings should be used.

One possible LHC scenario predicts $7 \mathrm{TeV}$ proton collisions with a luminosity of $\sim 10^{31} \mathrm{~cm}^{-2} \mathrm{~s}^{-1}$. In this scenario charm decays will be included in the list of BW-Channels as the trigger can be much less stringent. In this condition it is possible then to increase the L $0 \times \mathrm{HLT} 1$ prompt charm efficiency from about $15 \%$ to $70 \%$.

The L0 trigger has been commissioned using cosmic rays and muon showers produced by the Transition-line End Dump (TED) events during LHC injection tests. In order to commission the HLT a Full Experiment System Test (FEST) has been developed. It consists of a data-injector that feeds the event filter farm with simulated front-end electronic events so the full HLT can be exercised. The LHCb trigger farm size was chosen supposing that the average HLT1 event processing time should be less than $10 \mathrm{~ms}$. The HLT1 has been commissioned using FEST and the mean HLT1 processing time is of the order of $5 \mathrm{~ms}$.

\section{Conclusion}

The LHCb trigger is flexible and can accommodate different LHC running scenarios. The lowest trigger level has been commissioned using cosmic rays and TED events. The higher trigger levels have been exercised using FEST.

A set of BW-Channels presented in table 3 has been defined for the LHC nominal operation conditions and a procedure was established in order to setup the trigger for different running conditions. Based on Monte Carlo simulations and for nominal LHC conditions we expect to have 
trigger efficiencies in the rage of $70 \%$ to $90 \%$ for leptonic channels, from $20 \%$ to $50 \%$ for relevant hadronic channels and of the order of $50 \%$ for radiative B decays, as shown in table 4 . This will allow LHCb to collect $\sim 10^{9}$ fully reconstructed B-meson decays per $2 \mathrm{fb}^{-1}$, which is the annual expected luminosity at nominal conditions.

\section{References}

[1] The LHCb Collaboration, The LHCb Detector at the LHC, JINST 2008 JINST 3 S08005. 